\title{
Optimization of conditions of a heterogeneous catalytic reaction
}

\author{
S N Koledin ${ }^{1}$, K F Koledina ${ }^{1,2}$, I M Gubaydullin ${ }^{1,2}$ and A F Mullayanova ${ }^{2}$ \\ ${ }^{1}$ Ufa State Petroleum Technological University, Ufa, Bashkortostan, Russia, 450062 \\ ${ }^{2}$ Institute of Petrochemistry and Catalysis, Russian Academy of Sciences, Ufa, Bashkortostan, \\ Russia, 450075
}

\begin{abstract}
A kinetic model of the heterogeneous catalytic reaction of ethanol dehydrogenation to ethyl acetate is considered. The kinetic model is used to solve the problem of optimization of the reaction conditions taking into account the reactant adsorption and desorption on the solid catalyst.
\end{abstract}

\section{Introduction}

In the mathematical modeling of heterogeneous reactions, it is necessary to use kinetic equations as the Langmuir-Hinshelwood dependences or the law of acting surfaces $[1,2]$. These equations are reported in the scientific literature in relation to the development of kinetic models of, for example, benzene alkylation with ethylene [3] or ethylbenzene dehydrogenation [4].

In the ethanol dehydrogenation to ethyl acetate, bioethanol is used [5]. This is a green chemistry reaction. The target reaction product, ethyl acetate, is used as a solvent, in the production of paint and varnish materials and for other applications. This reaction proceeds to a high conversion and is highly selectivein the presence of copper-zinc-chromium catalysts [6]. The gas-phase reaction on transition metal catalysts is heterogeneous.

\section{Kinetics of ethanol dehydrogenation to ethyl acetate}

A kinetic study of ethanol dehydrogenation to ethyl acetate has been reported in [6]. The reaction scheme indicating the key reversible steps is presented (Table 1). The kinetic and adsorption parameters have been determined.

Table 1. Scheme of chemical transformations and kinetic equations for ethanol dehydrogenation to ethyl acetate.

\begin{tabular}{ll}
\hline Scheme of chemical transformations & Kinetic equations \\
\hline $\mathrm{C}_{2} \mathrm{H}_{5} \mathrm{OH}\left(\mathrm{X}_{1}\right) \leftrightarrow \mathrm{CH}_{3} \mathrm{CHO}\left(\mathrm{X}_{2}\right)+\mathrm{H}_{2}\left(\mathrm{X}_{3}\right)$ & $w(1)=k(1) * \theta(1)-k(3) * \theta(2) * \theta(3)$ \\
$\mathrm{C}_{2} \mathrm{H}_{5} \mathrm{OH}\left(\mathrm{X}_{1}\right)+\mathrm{CH}_{3} \mathrm{CHO}\left(\mathrm{X}_{2}\right) \leftrightarrow \mathrm{CH}_{3} \mathrm{COOC}_{2} \mathrm{H}_{5}\left(\mathrm{X}_{4}\right)++\mathrm{H}_{2}$ & $w(2)=k(2) * \theta(1) * \theta(2)-k(4) * \theta(4) * \theta(3)$ \\
$\left(\mathrm{X}_{3}\right)$ &
\end{tabular}

where $\mathrm{X}_{1}$ is ethanol $\mathrm{C}_{2} \mathrm{H}_{5} \mathrm{OH} ; \mathrm{X}_{2}$ is acetaldehyde $\mathrm{CH}_{3} \mathrm{CHO} ; \mathrm{X}_{3}$ is hydrogen $\mathrm{H}_{2} ; \mathrm{X}_{4}$ is ethyl acetate $\mathrm{CH}_{3} \mathrm{COOC}_{2} \mathrm{H}_{5} ; \theta(i)$ is the fraction of the catalyst surface occupied by $\mathrm{i}$-th component (1), (2), $\mathrm{i}=1, \ldots, 4 ; k(j)$ are the rate constants of the steps (the units of measurement depend on the order of the particular step); and $w(1), w(2)$ are the reaction rates.

$$
\theta^{*}=1-\sum_{i=1}^{4} \theta(i)
$$

where $\theta *$ is the unoccupied fraction of the catalyst surface at any instant of time. 


$$
\theta(i)=\frac{b(i) * \frac{x(i)}{V}}{1+\sum_{i=1}^{4} b(i) * \frac{x(i)}{V}},
$$

where $b(i)$ is the adsorption coefficient of $\mathrm{i}$-th component according to the mathematical model based on the Langmuir-Hinshelwood mechanism for adsorption of reaction components on a solid catalyst, $x(i)$ is the concentration of $\mathrm{i}$-th component, $[\mathrm{mol} / \mathrm{L}], V$ is the gas-phase volume of the reaction mixture, $\left[\mathrm{m}^{3}\right]$, which is determined from the geometric dimensions of the reactor [6].

The activation parameters for the rate constants and adsorption coefficients have been determined earlier [6].

The developed kinetic model of the complex catalytic reaction can be used to optimize the reaction conditions [7-13].

\section{Variable parameters and objective functions for optimization of conditions of the catalytic heterogeneous reaction of ethanol dehydrogenation to ethyl acetate}

The variable parameters of optimization as appliedto problems of chemical kinetics may include the temperature, type of the catalyst, concentration of the catalyst, pressure and so on. The experimental studies of this reaction were carried out at different temperatures and pressures. These parameters affect the volume of the reaction mixture. For the reaction in question, we take temperature and pressure as the variable parameters. The physico-chemical constraints have been reported previously [6].

In the general form, the optimization criterion based on the kinetic model has the form [14]

$$
R\left(\mathbf{x}, \mathbf{x}^{\mathbf{0}}, t^{*}, \boldsymbol{\eta}, \mu, T, P\right) \rightarrow \max
$$

where $x$ is the concentration vector of compounds, mol/L; $x_{0}$ is the vector of the initial concentrations of compounds, $\mathrm{mol} / \mathrm{L} ; \eta$ is the vector of compound weights; $\mu$ are additional expenses; $t^{*}$ is reaction time, min; $T$ is temperature, ${ }^{\circ} \mathrm{C}, P$ is pressure, atm.

The conditions of ethanol dehydrogenation to ethyl acetate were optimized according to relation (3) considering the following criteria reported previously [15].

1) Yield of the target product $x_{\text {prod }}$, which depends on the temperature and pressure:

$$
R_{1}(T, P)=x_{\text {prod }}(T, P) \rightarrow \max .
$$

The target product of the reaction is ethyl acetate $\left(\mathrm{X}_{4}\right)$.

2) Yield of the by-product $x_{b y-p r o d}$, which depends on the temperature and pressure:

$$
R_{2}(T, P)=x_{\text {by-prod }}(T, P) \rightarrow \min .
$$

The by-product of the reaction is acetaldehyde $\left(\mathrm{X}_{2}\right)$.

\section{Parallelization of the computational process}

Mathematical modeling of complex chemical reactions faces the following difficulties:

- there are several hypothetical reaction mechanisms and each of them should be addressed to choose the best one;

- several experiments carried out under different conditions are available (usually more than five); considering the existing experimental error, all of them should be addressedand the best two (or three) should be chosen, that is, those for which the calculated values coincide most closely with the experimental data;

- each of the kinetic parameters is determined ambiguously, being dependent on the correct choice of the initial approximation proceeding from some physicochemical assumptions.

The successive solution of these problems requires a lot of time (from several months to a year). It is proposed to arrange the problems in groups and carry out computations for these groups in parallel, with the computation within each group being carried out sequentially (Fig. 1) [16, 17]. 
The first group combines all of the mechanisms proposed for the given reaction (Stage 1). For each mechanism, all available experiments for this reaction are considered (Stage 2). For each experiment, the parametric plane is split to search for kinetic parameters (Stage 3).

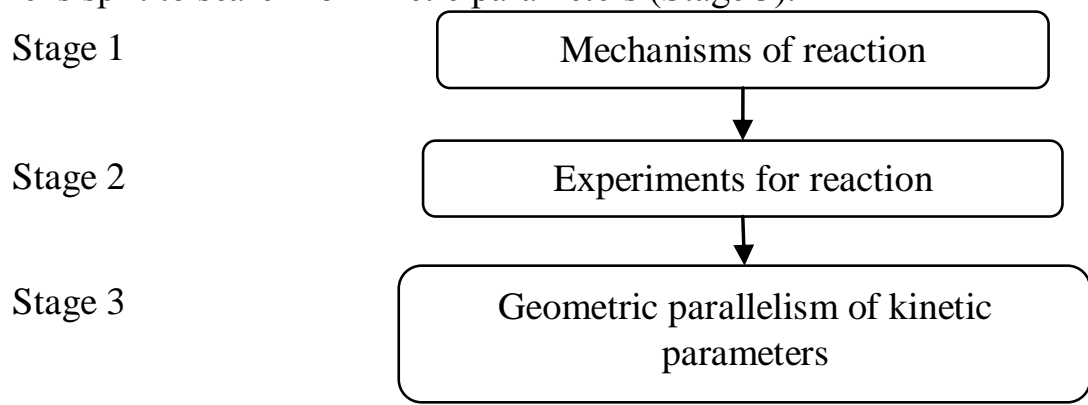

Figure 1. Model of parallelization of the computational process.

For large dimensions of the definition domain, population-based algorithms for solving the optimization problem are used. Parallelization models are employed for these algorithms [18]. The use of the genetic algorithm for solving the optimization problem implies the use of the following parallelization models:

1) Island Model

A multi-population is created as several subpopulations (islands), the number of which is equal to the number of processors used $S=Y_{i=l}^{|P|} S_{i} ;|S|=Y_{i=1}^{|P|}|S|_{i}$ (where $S$ is multi-population, $S_{i}$ are subpopulations (islands), $|\mathrm{P}|$ is the number of processors) (Fig. 2). Each island is treated by a separate processor. During a specified period of time, the subpopulations develop independently and, after that, the islands are synchronized using a special process, in which data exchange takes place.

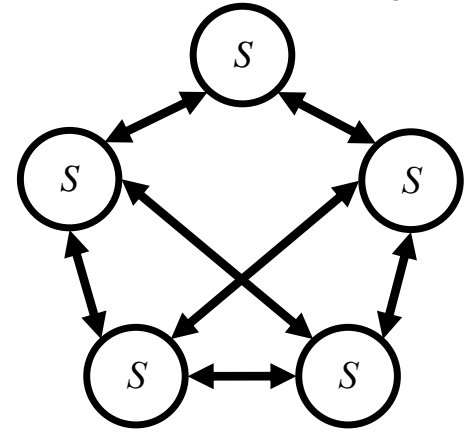

Figure 2. Island model of parallelization of population-based algorithms.

2) Cellular Model

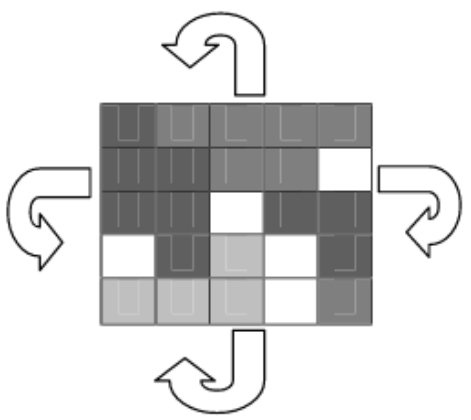

Figure 3. Cellular model of parallelization of population-based algorithms.

The area is split into parts according to the number of processors. The left-hand side is connected to the right-hand side and the upper side is connected to the lower side, thus forming a torus (Fig. 3). 
Each process can interact only with four neighbors (above, below, on the left, and on the right). Each cell contains only one solution (individual). Each process will choose the best individual among the neighbors, cross it over with the individual from its own cell, and place one offspring into its cell instead of the parent. The operation of this algorithm brings about effects resembling those in the island model. Initially, all individuals have random fitness (in Fig. 3, it is defined by colors). After several generations, relatively small regions of similar-fitness individuals are formed. As the algorithm operates, these regions grow and compete with one another.

3) Global Worker/Farmer model

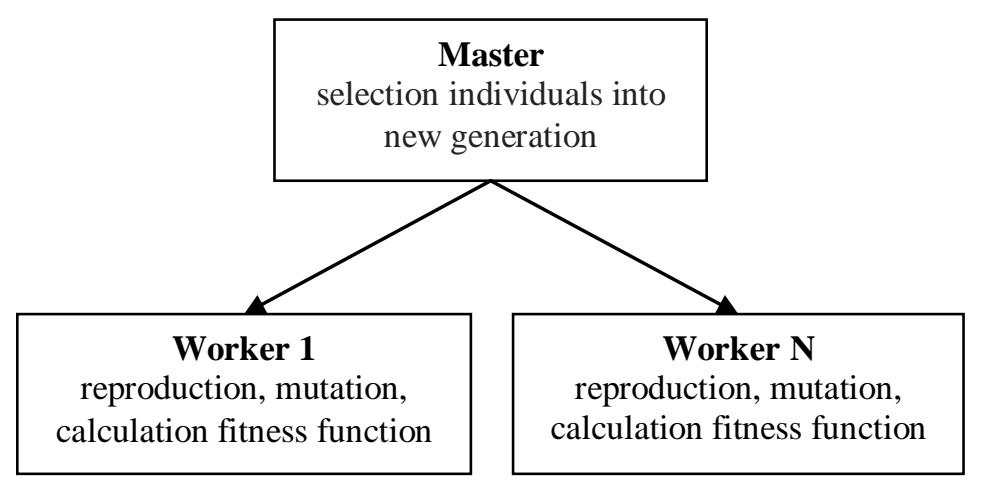

Figure 4. Global Worker/Farmer model of parallelization of population-based algorithms.

"Workers" (workstations) are responsible for reproduction, mutation, and calculation of the fitness function for selecting individuals to the new generation. All individuals created and evaluated by the "workers" are delivered to the "farmer" workstation, which then selects the individuals to the new population in conformity with fitness evaluation. The selected individuals are transferred by the "farmer" to the "workers" stations (Fig. 4).

The reaction conditions were optimized using the genetic algorithm to solve the optimization problem and the island model to parallelize the computational process [19].

The input processor receives a local range of variable parameters. The output processor givesout the optimal values for the variable parameters out of the indicated range. The values from this range are selected and used to solve the direct problem, that is, to solve a system of ordinary non-linear differential equations for determination of the objective functions. The optimal variable parameters for the given processor local range are found (Fig. 5).

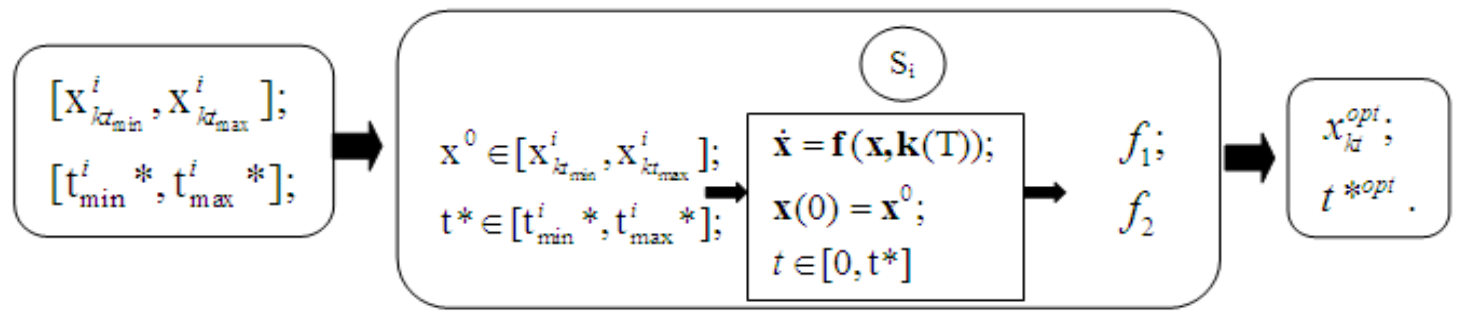

Figure 5. Treatment of the subpopulations by each processor of the island model of parallelization.

The optimized pressure and temperature for ethanol dehydrogenation to ethyl acetate were determined using the island model of parallelization. The time of the computational experiment was estimated (Fig. 6) (the calculations were carried out on a Intel Core I5 7th Gen quad-core PC).

The efficiency of utilizationof processors by the parallel algorithm for problem solution is defined by the relation

$$
E_{p}=\frac{S_{p}}{p}
$$


i.e., it is the mean fraction of algorithm execution time during which the processors are actually occupied by solution of the problem (Fig. 7).

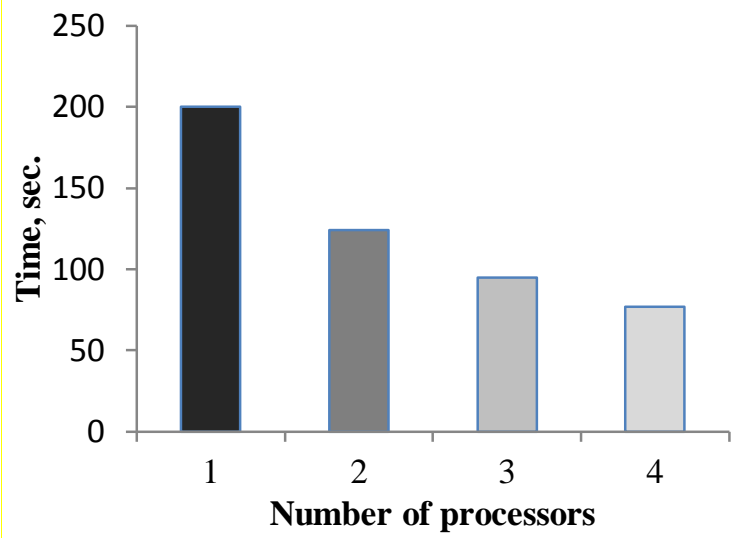

Figure 6. Time of the computational experiment with the genetic algorithm for different numbers of processors.

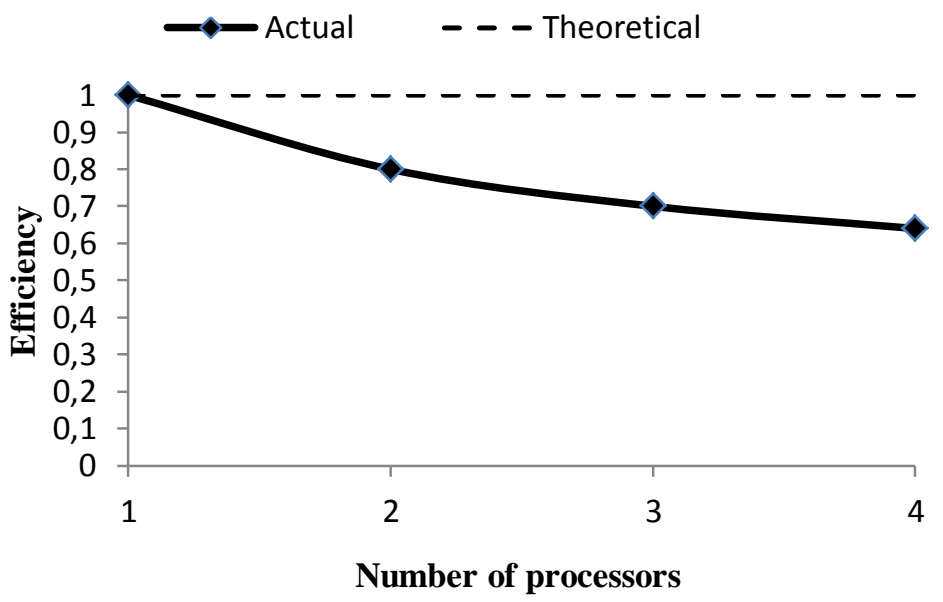

Figure 7. Efficiency of parallel program execution for the genetic algorithm.

The parallelization efficiency for the considered reactions was 65\%. Apparently, the ideal efficiency of parallelization is not achieved because of the time spent for data synchronization between the islands.

\section{Multiobjective optimization of the conditions of a catalytic heterogeneous reaction}

The major challenge in solving an optimization problem in chemistry is that all the theoretical works on optimization have addressed each criterion separately. However, in the last decades, numerous efficient evolutionary algorithms of multiobjective optimization have been proposed. These algorithms take into account all scientific experience in the approximation of the Pareto domination region and genetic algorithms. The computational power has markedly increased, which allows high-throughput computing to be accomplished over reasonable periods of time [20-27]. The multiobjective optimization involves the search for several Pareto-optimal solutions. The set of optimal values of variable parameters is the Pareto set. The objective functions in this set are called the Pareto front. The algorithm used most often to solve the multiobjective optimization problem is the NSGA-II algorithm $[22,23]$. According to this algorithm, the generated individuals are ranked, each one being assigned with a particular rank. The non-dominated points have the first rank, the points that are dominated only by first-rank points have the second rank, and so on. The crowding of the obtained individuals is also evaluated; the greater the distance between them, the higher the population diversity. In every 
iteration, the offsprings are selectedconsidering the rank and crowding (proximity) of individuals. Subsequently, the best points are chosen in the iteration via crossing over and mutation, which ensures the diversity of the next population. The parents and offsprings are combined into one population corresponding to the best solutions, and so on.

The problem was solved in the information system developed previously [28-31]. The optimization criteria include the yield of the target product and the yield of the by-product. The results of computational experiments are shown in Figs. 8-9.

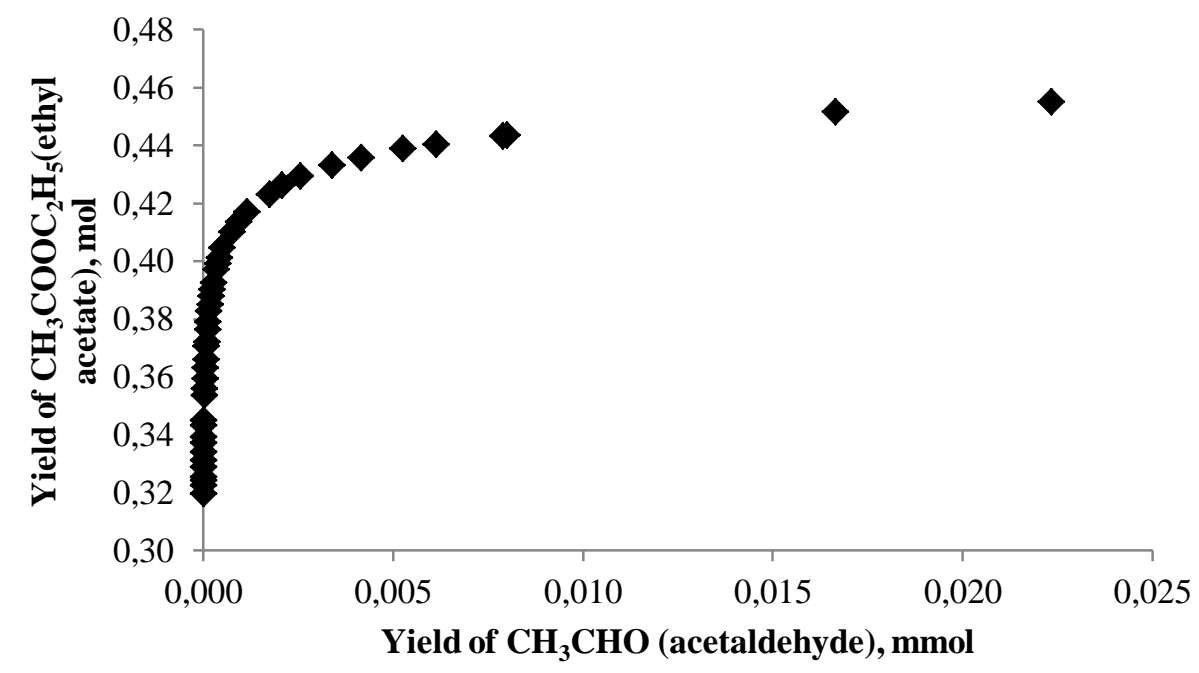

Figure 8. Pareto front approximation with the NSGA-II algorithm for ethanol dehydrogenation to ethyl acetate.

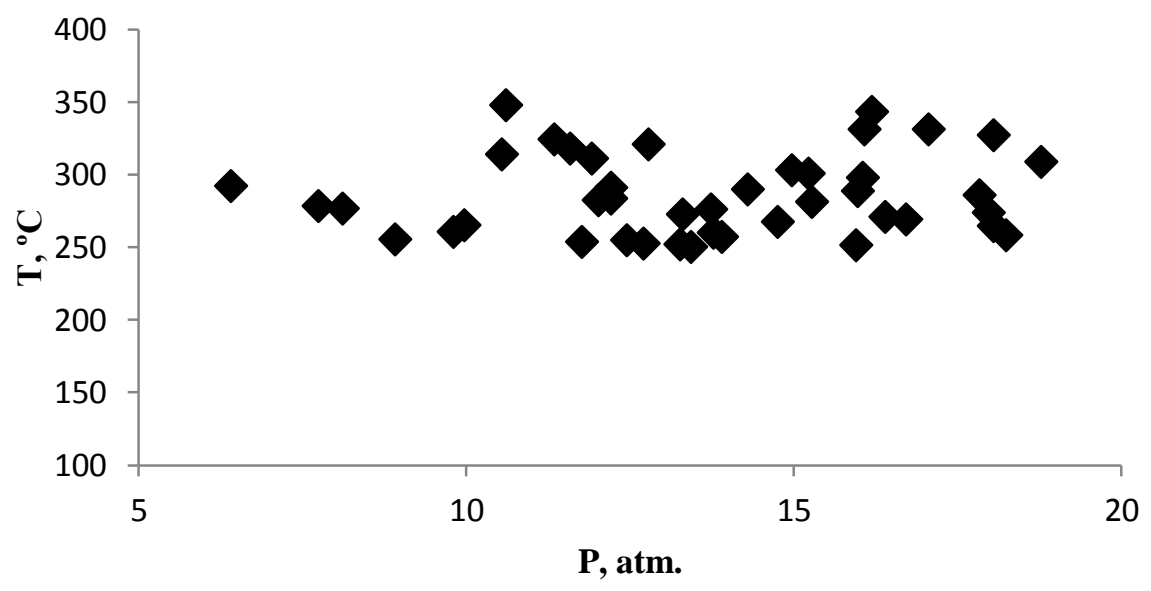

Figure 9. Pareto set approximation with the NSGA-II algorithm for ethanol dehydrogenation to ethyl acetate.

The resulting Pareto front and set approximations for ethanol dehydrogenation to ethylacetate allow the decision maker to choose the reaction conditions through the comparison of the Pareto set and front approximations for the corresponding objective functions (4) and (5) (Fig. 8, 9). Whenever it is necessary to maximize the yield of the target product (the target product is highly valuable) or minimize the yield of the by-product, the corresponding approximated values of the Pareto set and front can be used.

Thus, the optimal conditions for the complex heterogeneous catalytic reaction were studied by multiobjective optimization methods on the basis of a kinetic model of the reaction. 


\section{References}

[1] Slinko M 2007 Theoretical Foundations of Chemical Technology 41(1) 16-34

[2] Froment G 2005 Catal Rev Sci Eng 47(1) 83-124

[3] Inui K, Rurabayashi Tand Sato S 2002 J Catal 212(2) 125

[4] Bolder F 2008 Ind Eng Chem Res 47(19) 4796

[5] Elliott D and Pennella F 1989 J Catal 119359

[6] Men'Shchikov V, Gol'Dshtein L and Semenov I 2014 Kinetics and Catalysis 55(1) 12-17

[7] Spivak S, Koledina K, Koledin S and Gubaidullin I 2017 Journal of Applied Informatics 12(1) 39-49

[8] Koledina K, Koledin S and Gubaydullin I 2017 CEUR Workshop Proceedings 1966 5-9

[9] Gubaydullin I, Koledina K and Sayfullina L 2014 Eng J 18(1) 13-24

[10] Nurislamova L, Gubaydullin Iand Koledina K 2015 Reac Kinet Mech Cat 116(1) 79-93

[11] Nurislamova L, Gubaydullin I, Koledina K and Safin R 2016 Reac Kinet Mech Cat 117(1) 1-14

[12] Maskov D and Gubaidullin I 2012 Bulletin of Omsk University 64(2) 182-184

[13] Keil F 2013 Computers \& Mathematics with Applications 65(10) 1674

[14] Koledina K, Koledin S, Shchadneva N and Gubaidullin I 2017 Rus J Phys Chem A 91(3) 444449

[15] Koledina K, Koledin S, Schadneva N, Mayakova Y and Gubaydullin I 2017 Reac Kinet Mech Cat 121(2) 425-428

[16] Gubaydullin I, Lind Yu and Koledina K 2012 Num Methods Prog: Adv Comput 13 (1) 236-244

[17] Baynazarova N, Koledina K and Pichugina D 2016 CEUR Workshop Proceedings 1576 425431

[18] Awrejcewicz J, Lind Y, Gubaydullin I and Koledina K 2012 Theoretical and Applied Mechanics Letters 2(4) 043

[19] Koledina Kand Gubaidullin I 2013 Science and Education: a scientific edition of the MSTU N E Bauman 7 385-398

[20] Karpenko A 2016 Information and Mathematical Technologies in Science and Management 2 8-17

[21] Sobol I and Statnikov R 2006 The Choice of Optimal Parameters in Problems With Many Criteria: Textbook. Manual for Universities (Moscow: Drofa) p 175

[22] Deb K, Mohan M and Mishra S 2003 Evolutionary Multi-Criterion Optimization 2632 222-236.

[23] Kalyanmoy D, Pratap A, Agarwal S and Meyarivan T 2002 IEEE Transactions on Evolutionary Computation 6(2) 182-197

[24] Abramson M, Charles A, Dennis J, Digabel J and Digabel S 2009 SIAM Journal on Optimization 20(2) 948-966

[25] Alonso P, Argüeso F, Cortina R, Ranilla J and Vidal A 2013 J Math Chem 51(4) 1153-1163

[26] Alonso P, Argüeso F, Cortina R, Ranilla J and Vidal A 2012 J Math Chem 50(2) 410-420

[27] Canedo M and González-Hernández J 2011 J Math Chem 49(1) 163-184

[28] Vovdenko M, Gubaidulin I, Koledina K and Koledin S 2017 CEUR Workshop Proceedings $196620-23$

[29] Koledina K and Gubaidullin I 2016 Rus J Phys Chem A 90(5) 914-921

[30] Zainullin R, Koledina K, Akhmetov A and Gubaidullin I 2017 Kinetics and Catalysis 58(3) 279-289

[31] Koledin S, Koledina K, Gubaidullin I and Spivak S 2016 Chemical Industry today 10 24-35

\section{Acknowledgments}

The reported study was funded by RFBR according to the research projects № 18-07-00341, 18-3700015 and by the President of the Russian Federation SP-669.2018.5 stipends. 